Check for updates

Cite this: RSC Adv., 2017, 7, 48942

Received 7th August 2017

Accepted 13th October 2017

DOI: $10.1039 / c 7 r a 08731 d$

rsc.li/rsc-advances

\section{Investigation on the interaction of antibacterial drug moxifloxacin hydrochloride with human serum albumin using multi-spectroscopic approaches, molecular docking and dynamical simulation}

\author{
Xinnuo Xiong, Jiawei He, Hongqin Yang, Peixiao Tang, * Bin Tang, Qiaomei Sun \\ and Hui Li (D) *
}

\begin{abstract}
The interaction between moxifloxacin hydrochloride $(\mathrm{MOXH})$ and human serum albumin (HSA) was experimentally and simulatively investigated. Fluorescence quenching presented that MOXH bound to HSA via a static process, resulting in the formation of MOXH-HSA complex. This quenching mechanism was further verified by time-resolved fluorescence. Binding constants $\left(K_{\mathrm{a}}\right)$ of the complex were found to be $10^{5} \mathrm{~L} \mathrm{~mol}^{-1}$ according to fluorescence data, and the calculated thermodynamic parameters indicated that hydrogen bonds and van der Waals force played key roles in the binding process. The UV-vis absorption, synchronous fluorescence, three-dimensional fluorescence, and circular dichroism spectra suggested that binding with MOXH induced the conformational changes on HSA; the hydrophobicity around tryptophan residues increased, the $\alpha$-helix content increased, whereas the $\beta$-sheet and turn content of HSA decreased. Displacement experiments demonstrated that MOXH mainly bound to site I of HSA. Molecular docking results supported the active site and showed that the diazabicyclo of $\mathrm{MOXH}$ inserted into the hydrophobic pocket of HSA. Molecular dynamics simulation further ascertained that MOXH steadily bound to site I of HSA. In conclusion, hydrogen bonds and VDW force played major roles in stabilizing the MOXH-HSA complex, and hydrophobic force was also involved in the binding process.
\end{abstract}

\section{Introduction}

Moxifloxacin hydrochloride (MOXH) is a novel fourthgeneration quinolone antimicrobial drug with fewer side effects and an extended half-life. Compared with older fluoroquinolones, MOXH has enhanced activity against Grampositive and atypical bacteria, while retaining Gram-negative activity., ${ }^{1,2}$ Several studies have shown that MOXH can treat bacterial conjunctivitis and a wide variety of bacterial infections including pneumonia, sinusitis, and worsening of chronic bronchitis. ${ }^{1,3-5}$ Furthermore, it has been widely used in the treatment and prevention of veterinary diseases in animals intended for human consumption and commercially farmed fish. ${ }^{6}$

Studies on the interaction between drugs and biological macromolecules can provide a proper understanding of the absorption, transportation, distribution, metabolism, excretion properties and possible mechanism of drugs, thereby are beneficial to design, modify and screen drug molecules. ${ }^{7,8}$

College of Chemical Engineering, Sichuan University, Chengdu, Sichuan, China. E-mail: tangpeixiao@126.com; lihuilab@sina.com; Fax: +86028 85401207; Tel: +8602885405149
Previously, the interactions of MOXH with biological macromolecules including calf thymus $\mathrm{DNA}^{2,9}$ and bovine hemoglobin ${ }^{3}$ have been investigated by fluorescence spectroscopy. These studies indicated that MOXH could bind to the two biological macromolecules through a static process and affect their structures via different combination types. Thus, the binding of MOXH to biological macromolecules may influence the function of macromolecules and the drug efficacy. However, to our knowledge, a comprehensive study on the interaction between MOXH and the major protein in the human blood circulation remains unclear. Among these proteins, human serum albumin (HSA) is the most abundant in blood plasma. HSA regulates the transportation of various drugs and helps maintain the osmotic pressure of blood. ${ }^{10,11}$ Drug-HSA interaction plays a significant role in understanding the pharmacokinetics and pharmacological effects of drugs. ${ }^{12,13}$ Hence, pharmacokinetic studies should be conducted in order to ascertain MOXH's presence in the blood plasma, especially with regards to its transportation and distribution properties in the circulatory system after oral administration.

In the present study, attempts were conducted to assess the binding process of MOXH with HSA by employing multispectroscopic techniques including fluorescence, time-resolved 
fluorescence, UV-vis absorption spectroscopy, and circular dichroism spectroscopy. The binding information such as quenching mechanism, binding parameters, acting forces, binding mode and conformational changes were discussed. Moreover, molecular docking and dynamics simulations were applied to evaluate the dynamics and stability of the MOXH-HSA complex, and to corroborate the results obtained from spectroscopy studies.

\section{Experimental}

\subsection{Materials}

MOXH (99\% purity) was purchased from Yitai Technology Co. (Shanghai, China). Fatty-acid-free HSA (A1887-5G) was acquired from Sigma Aldrich (St. Louis, MO, USA). Phenylbutazone and ibuprofen were purchased from J\&K Scientific Ltd. (Beijing, China). The stock solution of HSA was prepared at $2.0 \times$ $10^{-5} \mathrm{~mol} \mathrm{~L}^{-1}$ in phosphate buffer solution (PBS, $\mathrm{pH}=7.4$ ). MOXH stock solution was prepared at a concentration of $2.0 \times$ $10^{-4} \mathrm{~mol} \mathrm{~L}^{-1}$ with PBS. All solutions were diluted to the required volume with PBS prepared by triple-distilled water. All stock solutions were stored at $0-4{ }^{\circ} \mathrm{C}$.

\subsection{Fluorescence spectroscopy measurements}

Fluorescence analysis was executed on a Cary Eclipse fluorescence spectrophotometer (Varian, CA, USA) equipped with $1.0 \mathrm{~cm}$ quartz cells. The HSA concentration was fixed at $2.0 \times$ $10^{-6} \mathrm{~mol} \mathrm{~L}^{-1}$ while the MOXH concentrations varied from 0 to $7.0 \times 10^{-6} \mathrm{~mol} \mathrm{~L}^{-1}$. Prior to fluorescence measurements, samples were thermally equilibrated at $298 \mathrm{~K}, 304 \mathrm{~K}$, and $310 \mathrm{~K}$ for about $30 \mathrm{~min}$. An excitation wavelength of $280 \mathrm{~nm}$ was employed, and the emission spectra were recorded from $290 \mathrm{~nm}$ to $450 \mathrm{~nm}$. The widths of excitation and emission slits were set at 5 and $10 \mathrm{~nm}$, respectively. To eliminate inner filter effect, fluorescence intensities were corrected based on the following formula: ${ }^{14,15}$

$$
F_{\text {corr }}=F_{\text {obs }} \times \mathrm{e}^{\frac{A_{\text {ex }}+A_{\text {em }}}{2}}
$$

where $F_{\text {corr }}$ and $F_{\text {obs }}$ are the corrected and measured fluorescence intensities, respectively; $A_{\mathrm{ex}}$ and $A_{\mathrm{em}}$ are the absorbance values at excitation and emission wavelengths, respectively.

Synchronous fluorescence spectra of HSA with various MOXH concentrations were recorded in the wavelength range of 200-400 $\mathrm{nm}$ at $298 \mathrm{~K}$. The excitation and emission wavelength interval $(\Delta \lambda)$ were set at 15 and $60 \mathrm{~nm}$, respectively.

Three-dimensional (3D) fluorescence spectra of HSA $(2.0 \times$ $10^{-6} \mathrm{~mol} \mathrm{~L}^{-1}$ ) and the HSA-MOXH mixture solution (molar ratio, $1: 1$ ) were obtained at the excitation wavelength range from 200 to $400 \mathrm{~nm}$ with $5 \mathrm{~nm}$ increments. The emission spectra were monitored between 200 and $500 \mathrm{~nm}$.

\subsection{Time-resolved fluorescence spectra}

Time-resolved fluorescence measurements were conducted by time-correlated single-photon counting technique with a Horiba Jobin Yvon FluoroLog-TCSPC spectrofluorometer (HORIBA, Les Ulis, France). The HSA concentration was $2.0 \times$
$10^{-6} \mathrm{~mol} \mathrm{~L}^{-1}$, and the MOXH concentration varied from 0 to 5.0 $\times 10^{-6} \mathrm{~mol} \mathrm{~L}^{-1}$. Samples were recorded by fixing $280 \mathrm{~nm}$ as the excitation wavelength and $337 \mathrm{~nm}$ as the emission wavelength at room temperature.

\subsection{UV-vis spectroscopy}

Absorbance measurements were performed at $298 \mathrm{~K}$ using a TU1901 spectrophotometer (Purkinje General, Beijing, China) from $200 \mathrm{~nm}$ to $500 \mathrm{~nm}$. The absorption spectra were obtained at constant HSA concentration $\left(2.0 \times 10^{-6} \mathrm{~mol} \mathrm{~L}^{-1}\right)$ and varying MOXH concentrations from 0 to $7.0 \times 10^{-6} \mathrm{~mol} \mathrm{~L}^{-1}$.

\subsection{Circular dichroism (CD) spectroscopy}

CD spectra were collected on a fluorescence spectrometer (Hitachi-F7000, Tokyo, Japan) equipped with R3788 photomultiplier at $298 \mathrm{~K}$. The measurements were performed by fixing the concentration of HSA constant $\left(2.0 \times 10^{-6} \mathrm{~mol} \mathrm{~L}^{-1}\right)$, and varying the MOXH concentration from 0 to $5.0 \times$ $10^{-6} \mathrm{~mol} \mathrm{~L}^{-1}$ ([MOXH] : [HSA] $=0: 1,1: 1$, and $\left.2.5: 1\right)$. Each spectrum was obtained from $190 \mathrm{~nm}$ to $250 \mathrm{~nm}$ with $1 \mathrm{~nm}$ step size, $1 \mathrm{~nm}$ band width, and $0.5 \mathrm{~s}$ average time. An average of three scans was obtained for all spectra. The data were expressed as mean residue ellipticity (MRE; $\mathrm{deg} \mathrm{cm}^{2} \mathrm{dmol}^{-1}$ ) and were calculated using the following equation:

$$
\mathrm{MRE}=\frac{\text { observed CD }(\mathrm{m} \mathrm{deg})}{10 C_{\mathrm{p}} n l}
$$

where $C_{\mathrm{p}}$ is the molar concentration of the protein, $n$ is the number of amino acid residues, and $l$ is the path length.

\subsection{Site marker displacement studies}

Displacement experiments were conducted using the site markers, phenylbutazone and ibuprofen. The molar ratio of MOXH to HSA was maintained at $1: 1([\mathrm{HSA}]=2.0 \times$ $10^{-6} \mathrm{~mol} \mathrm{~L}^{-1}$ ). The binary MOXH-HSA solutions were vortexmixed and incubation for $30 \mathrm{~min}$. Then, the phenylbutazone and ibuprofen solutions with varied concentrations were gradually added and incubated for another $30 \mathrm{~min}$. Fluorescence spectra were recorded at $298 \mathrm{~K}$ under the same experimental conditions as previously described.

\subsection{Molecular docking}

Binding mode of the ligand with HSA was examined by molecular docking using the FlexX program ${ }^{\mathbf{1 6}}$ interfaced within LeadIT. The 3D structure of MOXH and HSA were downloaded from PubChem (PubChem CID: 152946) and RCSB protein data bank (PDB: 1H9Z), respectively. A global docking including all amino acid residues of HSA was performed. Correct tautomers and protonation states were assigned, and hydrogen atoms were added to the binding site residues using the receptor wizard. The FlexX hybrid algorithm (enthalpy and entropy driven ligand binding), as available in LeadIT, ${ }^{17}$ was used to place the "base fragment". Other parameters were set at their default values. 


\subsection{Molecular dynamics (MD) simulations}

MD simulations were conducted with YASARA v17.4.17, ${ }^{18}$ using the AMBER14 force field. ${ }^{19}$ Partial atomic charges of ligand were computed using the AM1-BCC model ${ }^{20}$ implemented in YASARA. MD simulations in explicit water were performed at a constant temperature $(298.15 \mathrm{~K})$ after initial energy minimization procedures. Periodic boundary conditions were applied to system, and counter ions were randomly added by replacing water molecules with $\mathrm{Na}$ or $\mathrm{Cl}$ to provide a charge-neutral system. Longrange Coulomb interactions were included using particle-mesh Ewald (PME) summation and a cut-off of 8.0 Å. Simulations were carried out in their entirety, employing a pre-defined macro (md run) within the YASARA package. Multiple time steps were used in the simulation: 1.25 fs for intramolecular and 2.5 fs for intermolecular forces, and data were collected every $100 \mathrm{ps}$. The two-dimensional maSp of interaction were calculated using LigPlot program. ${ }^{21}$

\section{Results and discussion}

\subsection{Quenching mechanism}

Fluorescence spectroscopy is widely used to study drug-protein interactions due to its exceptional sensitivity, selectivity, convenience, and abundance of theoretical foundation. ${ }^{22}$ The effect of MOXH on the fluorescence intensity of HSA at $298 \mathrm{~K}$ was displayed in Fig. 1(a). HSA generated an obvious fluorescence emission band at $337 \mathrm{~nm}$, which was gradually quenched with increasing MOXH concentrations. In addition, MOXH alone did not emit fluorescence at the highest concentration. Therefore, such a decrease in intensity can be ascribed to the quenching of HSA by MOXH.

The quenching mechanism is frequently categorized into dynamic quenching, static quenching, or a combination of both two, which can be distinguished by their differential dependence on temperature. ${ }^{23}$ Given that dynamic quenching depends on diffusion and high temperatures to achieve larger diffusion coefficients, the quenching constant should increase with temperature risen. However, the effect is typically reversed in static quenching. That is, increased temperatures are likely to decrease the quenching constant because of the stability of ground state complexes were affected by temperature. ${ }^{\mathbf{2 4 , 2 5}}$ To explore the quenching mechanism qualitatively, the fluorescence data at different temperatures were analyzed using the Stern-Volmer equation: ${ }^{26}$
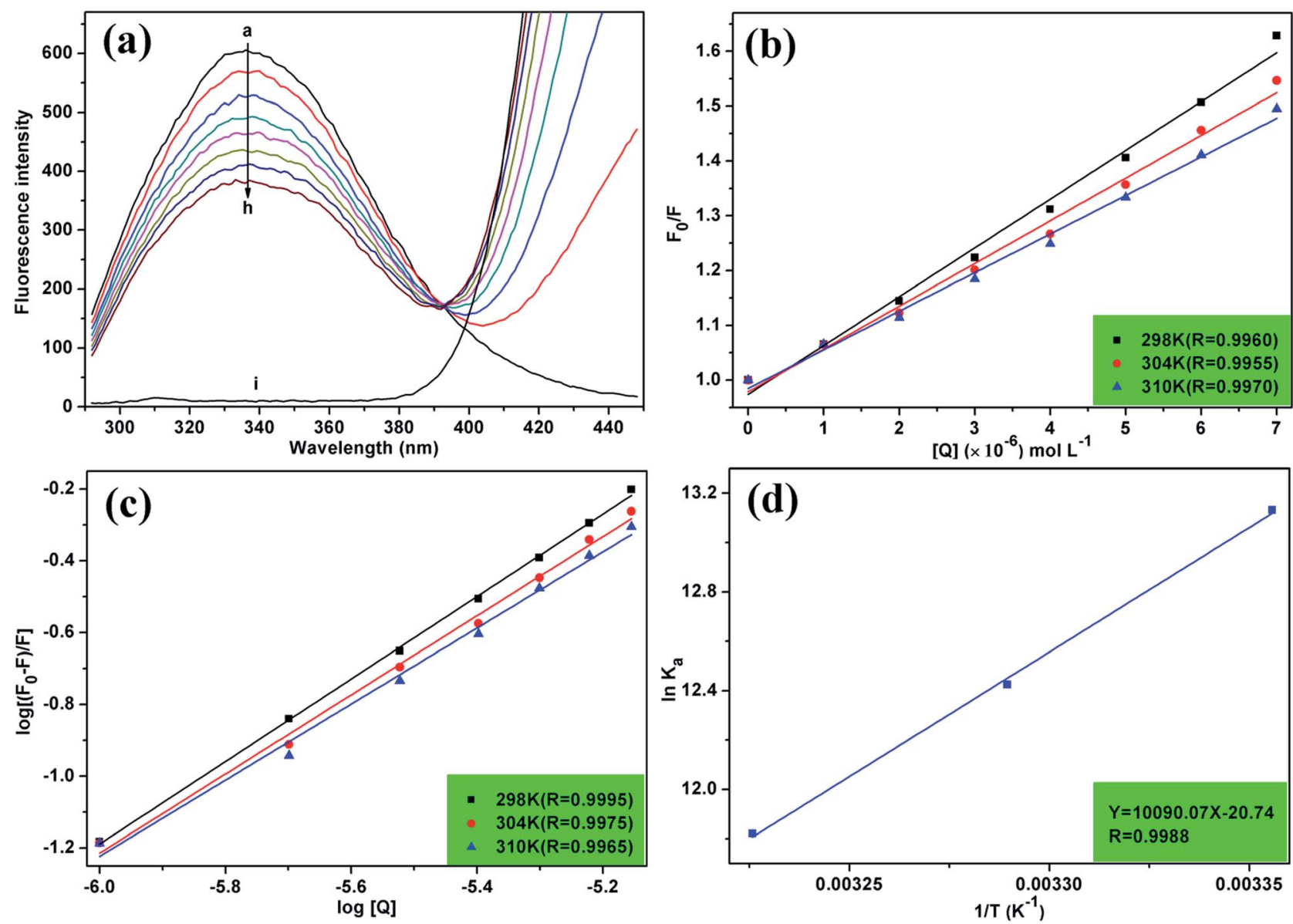

Fig. 1 (a) Fluorescence spectra of HSA $\left(2.0 \times 10^{-6} \mathrm{~mol} \mathrm{~L}^{-1}\right)$ in the absence and presence of MOXH at $298 \mathrm{~K}$. MOXH concentrations (a-h) were 0 , $1.0,2.0,3.0,4.0,5.0,6.0$, and $7.0 \times 10^{-6} \mathrm{~mol} \mathrm{~L}^{-1}$. Curve $i$ shows the emission spectrum of MOXH alone $\left.\left(7.0 \times 10^{-6} \mathrm{~mol} \mathrm{~L}\right)^{-1}\right) .(\mathrm{b}) \mathrm{Stern}^{-\mathrm{Volmer}}$ plots for MOXH interacting with HSA at three different temperatures. (c) Plot of log $\left[\left(F_{0}-F\right) / F\right]$ versus log $[Q]$ for the MOXH-HSA system at three different temperatures. (d) van't Hoff plot of the MOXH-HSA system. 
Table 1 Stern-Volmer quenching constants, binding parameters, and thermodynamic parameters of MOXH-HSA system at different temperatures

\begin{tabular}{|c|c|c|c|c|c|c|}
\hline \multirow[b]{2}{*}{$T(\mathrm{~K})$} & \multirow{2}{*}{$\begin{array}{l}\begin{array}{l}\text { Stern-Volmer } \\
\text { quenching constants }\end{array} \\
K_{\mathrm{SV}} \times 10^{4}\left(\mathrm{~L} \mathrm{~mol}^{-1}\right)\end{array}$} & \multicolumn{2}{|l|}{ Binding parameters } & \multicolumn{3}{|c|}{ Thermodynamic parameters } \\
\hline & & $K_{\mathrm{a}} \times 10^{5}\left(\mathrm{~L} \mathrm{~mol}^{-1}\right)$ & $n$ & $\Delta G\left(\mathrm{~kJ} \mathrm{~mol}{ }^{-1}\right)$ & $\Delta H\left(\mathrm{~kJ} \mathrm{~mol}^{-1}\right)$ & $\Delta S\left(\mathrm{~J} \mathrm{~mol}^{-1} \mathrm{~K}^{-1}\right)$ \\
\hline 298 & 8.901 & 5.407 & 1.148 & -32.34 & -80.89 & -172.97 \\
\hline 304 & 7.794 & 2.489 & 1.101 & -31.30 & & \\
\hline 310 & 7.041 & 1.361 & 1.059 & -30.60 & & \\
\hline
\end{tabular}

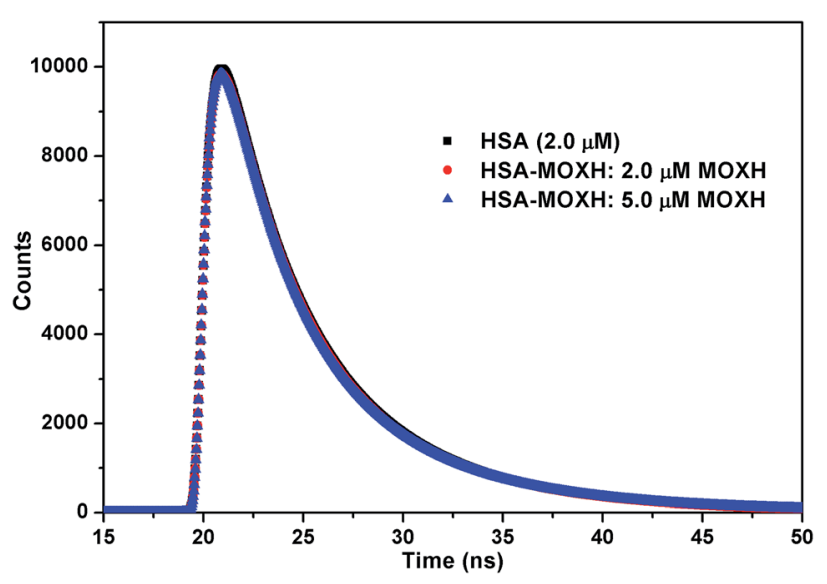

Fig. 2 Time-resolved fluorescence lifetime of HSA in the absence and presence of various concentrations of $\mathrm{MOXH}$.

$$
\frac{F_{0}}{F}=1+K_{\mathrm{Sv}}[Q]
$$

where $F_{0}$ and $F$ are the fluorescence intensities in the absence and presence of a quencher, respectively; $K_{\mathrm{SV}}$ is the SternVolmer quenching constant and $[Q]$ is the quencher concentration. The $K_{\mathrm{SV}}$ can be determined using the linear regression of the plot of $F_{0} / F$ against [Q], as shown in Fig. 1(b). The calculated $K_{\mathrm{SV}}$ values at 298,304 , and $310 \mathrm{~K}$ are summarized in Table 1 . The $K_{\mathrm{Sv}}$ values decreased with increasing temperature, which indicated that the quenching of HSA by MOXH was initiated by complex formation via the static quenching process rather than dynamic collision. ${ }^{27}$

To further substantiate the quenching mechanism, fluorescence lifetime measurements were performed. Fig. 2 depicts the fluorescence decay of HSA in the absence and presence of MOXH. Evidently, the fluorescence decay profiles almost remained unchanged after the addition of MOXH at three different concentrations. The corresponding data were analyzed using tail fitting method, and the goodness of fit was assessed by $\chi^{2}$ values. The average fluorescence lifetime $(\tau)$ was calculated based on the decay time and the relative amplitude $(\alpha)$ by using the following equation:

$$
\tau=\sum \alpha_{i} \tau_{i}
$$

As presented in Table 2, the binding of MOXH did not cause an evident decrease in the $\tau$ values. In general, a decrease in the mean decay time of the entire excited-state population should be attributed to dynamic quenching; static quenching with the ground-state complex formed has almost no effect on the decay time of the uncomplexed fluorophores. ${ }^{28,29}$ Therefore, the static quenching mode between MOXH and HSA was further confirmed.

\subsection{Binding constants and the number of binding sites}

For static quenching, the binding constant $\left(K_{\mathrm{a}}\right)$ and the number of binding sites $(n)$ can be determined by plotting the doublelogarithm regression curve of the fluorescence data with the modified Stern-Volmer equation: ${ }^{12}$

$$
\log \frac{F_{0}-F}{F}=\log K_{\mathrm{a}}+n \log [Q]
$$

$K_{\mathrm{a}}$ and $n$ values were obtained from the intercept and slope of the curve of $\log \left[\left(F_{0}-F\right) / F\right]$ versus $\log [Q]$ [Fig. 1(c)], and were summarized in Table 1 . All values of $n$ were approximately equal to one, which indicated that a single high-affinity binding site existed in HSA. Moreover, MOXH exhibited a relatively strong affinity to HSA according to the $K_{\mathrm{a}}$ values $\left(10^{5}\right.$ magnitude).

\subsection{Thermodynamic parameters and the nature of the binding forces}

Four non-covalent interaction forces, including hydrogen bond, van der Waals (VDW) force, hydrophobic force, and electrostatic

Table 2 Fluorescence decay fitting parameters for the MOXH-HSA system with different concentrations of MOXH

\begin{tabular}{llllllll}
\hline Sample & $\tau_{1}(\mathrm{~ns})$ & $\tau_{2}(\mathrm{~ns})$ & $\tau_{3}(\mathrm{~ns})$ & $\alpha_{1}$ & $\alpha_{2}$ & $\alpha_{3}$ & $\tau(\mathrm{ns})$ \\
\hline Free HSA $(2.0 \mu \mathrm{M})$ & 3.211 & 0.584 & 6.918 & 0.344 & 0.041 & 0.615 & 5.383 \\
HSA-MOXH $(+2.0 \mu \mathrm{M})$ & 3.152 & 0.500 & 6.877 & 0.339 & 0.039 & 0.622 & 5.366 \\
HSA-MOXH $(+5.0 \mu \mathrm{M})$ & 3.095 & 0.438 & 6.839 & 0.338 & 0.038 & 0.624 & 5.330
\end{tabular}


interactions, promote small molecules binding to biological macromolecules. ${ }^{30}$ To characterize the binding force involved in MOXH-HSA, the thermodynamic parameters such as enthalpy change $(\Delta H)$, entropy change $(\Delta S)$, and Gibbs free energy change $(\Delta G)$, were calculated according to the van't Hoff equations: ${ }^{12,31}$

$$
\begin{aligned}
& \log K_{\mathrm{a}}=-\frac{\Delta H}{R T}+\frac{\Delta S}{R} \\
& \Delta G=\Delta H-T \Delta S
\end{aligned}
$$

where $K_{\mathrm{a}}$ is the binding constant at the corresponding temperature $(T)$, and $R$ is the gas constant. $\Delta H$ and $\Delta S$ values were obtained from the slope and intercept of the linear plot ln $K_{\mathrm{a}}$ versus $1 / T$ based on eqn (6) [Fig. 1(d)], and $\Delta G$ values were estimated from eqn (7) at corresponding temperatures (Table 1). The negative values for $\Delta G$ indicated that the binding of MOXH to HSA occurred spontaneously. The negative $\Delta H$ suggested that the binding reaction was an exothermic process, thereby providing a reasonable explanation for the decreasing $K_{\mathrm{a}}$ in Table 1 . Besides, the negative values of $\Delta H$ and $\Delta S$ demonstrated that hydrogen bonds and VDW force were the main forces governing binding in the MOXH-HSA system, according to the theory of Ross and Subramanian. ${ }^{30}$

\subsection{Investigation on the conformational changes of HSA}

3.4.1. UV-vis spectroscopy. UV-vis spectroscopy is a simple and intuitive approach to investigating the structural changes in protein-ligand interactions, similar to fluorescence spectra. The UV-vis spectra of HSA with various concentrations of MOXH were shown in Fig. 3(a). The absorption peak of HSA at about $280 \mathrm{~nm}$ increased with gradual addition of MOXH and a distinct red shift (from $279 \mathrm{~nm}$ to $287 \mathrm{~nm}$ ) was observed. This phenomenon indicated that MOXH bound to HSA which caused conformational changes of HSA, and thus the hydrophobicity around the aromatic amino acid residues increased. ${ }^{32}$

3.4.2. Synchronous fluorescence spectroscopy. Synchronous fluorescence spectroscopy can provide information about the microenvironment of the chromophore molecules. ${ }^{33}$ When $\Delta \lambda$ between excitation and emission wavelengths is stabilized at 15 and $60 \mathrm{~nm}$, shifts in the maximum emission of the synchronous fluorescence reflect the changes in polarity around
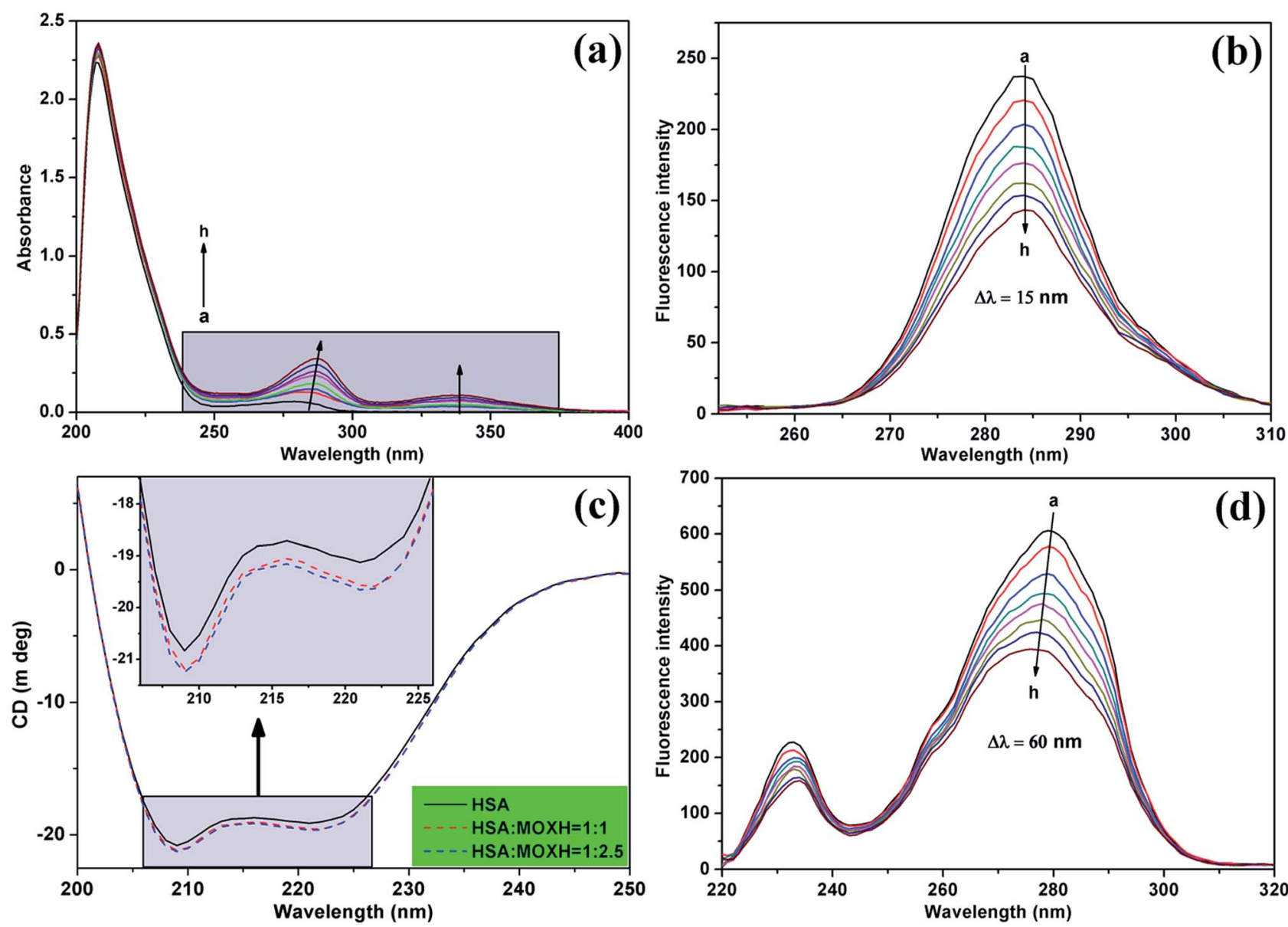

Fig. 3 (a) UV-vis absorption spectra of HSA $\left(2.0 \times 10^{6} \mathrm{~mol} \mathrm{~L}{ }^{-1}\right)$ with various concentrations of MOXH. Synchronous fluorescence spectra of HSA $\left(2.0 \times 10^{-6} \mathrm{~mol} \mathrm{~L}^{-1}\right)$ in the presence of varying concentrations of $\mathrm{MOXH}$ for (b) $\Delta \lambda=15 \mathrm{~nm}$ and (d) $\Delta \lambda=60 \mathrm{~nm}$. MOXH concentrations from "a" to " $h$ " in figures (a), (b), and (d) were $0,1.0,2.0,3.0,4.0,5.0,6.0$, and $7.0 \times 10^{-6} \mathrm{~mol} \mathrm{~L}^{-1}$, respectively. (c) Circular dichroism spectra of HSA (2.0 $\times$ $10^{-6} \mathrm{~mol} \mathrm{~L}^{-1}$ ) in the absence and presence of $\mathrm{MOXH}$. 
the tyrosine (Tyr) and tryptophan (Trp) residues of HSA, respectively. ${ }^{34}$ Fig. $3(\mathrm{~b})$ and (d) exhibit the synchronous fluorescence spectra of MOXH-HSA system. The fluorescence intensity of HSA regularly decreased with addition of $\mathrm{MOXH}$, which revealed the occurrence of fluorescence quenching during the binding process consistent with fluorescence data above. Furthermore, no shift occurred in the maximum emission wavelength at $\Delta \lambda=15 \mathrm{~nm}$ whereas a slight blue shift at $\Delta \lambda$ $=60 \mathrm{~nm}$ was observed. This phenomenon indicated that the MOXH binding affected the conformation of HSA and lead to an increase in hydrophobicity around the Trp residues, which was in line with the UV-vis result. ${ }^{12,24}$ To further clarify the secondary structural changes in HSA by MOXH, 3D and CD spectra studies were conducted.

3.4.3. 3D fluorescence spectroscopy. 3D fluorescence spectroscopy is known to provide detailed information regarding to the conformation of protein. ${ }^{35}$ Fig. 4 displays the 3D fluorescence spectra of HSA with and without MOXH. The related spectra parameters are listed in Table 3. As shown in Fig. 4, peak a represents the Rayleigh scattering peak $\left(\lambda_{\mathrm{ex}}=\lambda_{\mathrm{em}}\right)$. Peak I exhibits the spectral behavior of Trp and Tyr residues, corresponding to the microenvironment polarity; peak II mainly reveals the polypeptide backbone structures of HSA. ${ }^{36}$ After the addition of $\mathrm{MOXH}$, the fluorescence intensity of both peaks I and II showed an evident decrease. Furthermore, blue shift was
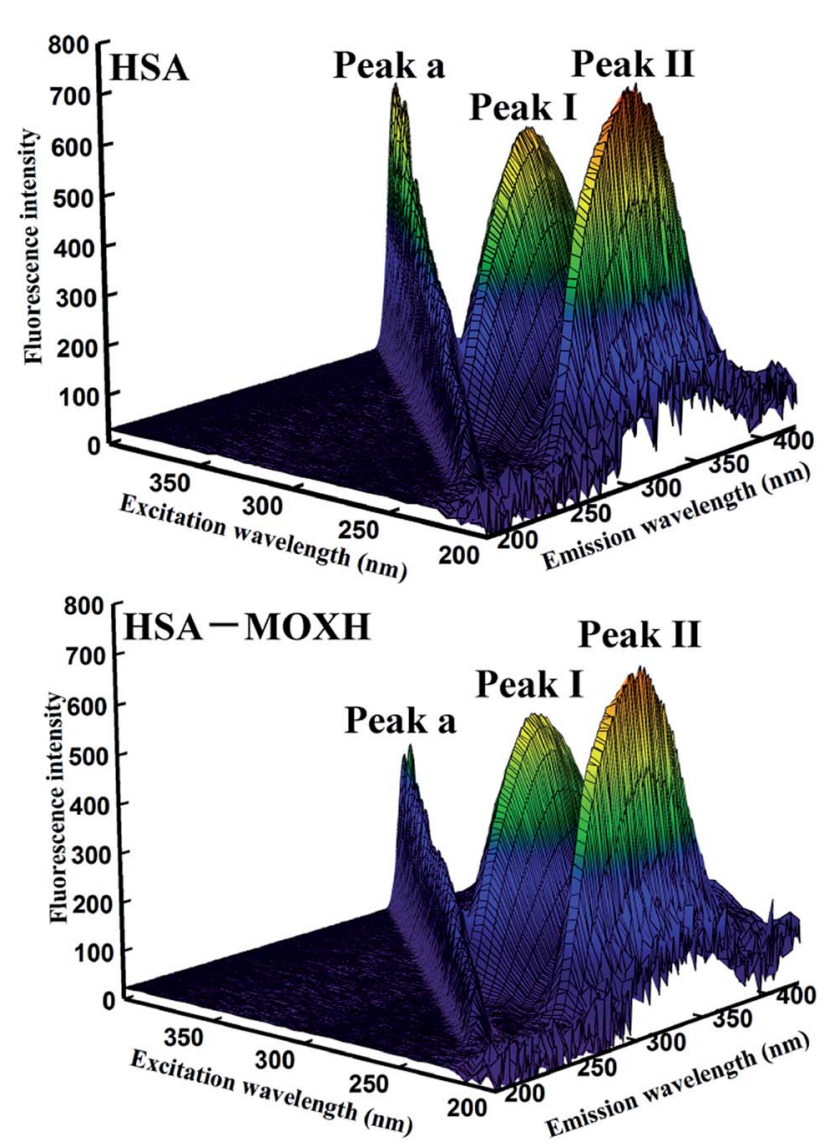

Fig. 4 3D fluorescence spectral of HSA $\left(2.0 \times 10^{-6} \mathrm{~mol} \mathrm{~L}^{-1}\right)$ in the absence and presence of MOXH $\left(2.0 \times 10^{-6} \mathrm{~mol} \mathrm{~L}^{-1}\right)$ at $298 \mathrm{~K}$.
Table 3 3D fluorescence spectral parameters of HSA in the absence and presence of $\mathrm{MOXH}$ at $298 \mathrm{~K}$

\begin{tabular}{llll}
\hline System & Peak position & \\
\hline HSA & Peak no. & $\begin{array}{l}\text { Peak } \\
{\left[\lambda_{\text {ex }} / \lambda_{\text {em }}(\mathrm{nm} / \mathrm{nm})\right]}\end{array}$ & Intensity \\
& A & $280 / 280 \rightarrow 350 / 350$ & $261.96 \rightarrow 449.80$ \\
MOXH-HSA & I & $280 / 338$ & 619.47 \\
& II & $225 / 336$ & 742.33 \\
& I & $280 / 280 \rightarrow 350 / 350$ & $119.95 \rightarrow 231.84$ \\
& II & $280 / 334$ & 569.71 \\
& & $225 / 329$ & 688.31
\end{tabular}

Table 4 Secondary structure composition of HSA in the presence of $\mathrm{MOXH}$ with different concentrations

\begin{tabular}{lllll}
\hline System & $\begin{array}{l}\alpha \text {-helix } \\
(\%)\end{array}$ & $\begin{array}{l}\beta \text {-sheet } \\
(\%)\end{array}$ & $\begin{array}{l}\text { Turn } \\
(\%)\end{array}$ & $\begin{array}{l}\text { Unordered } \\
(\%)\end{array}$ \\
\hline Free HSA & 46.3 & 14.0 & 16.9 & 25.4 \\
HSA : MOXH $=1: 1$ & 53.5 & 8.2 & 15.6 & 25.4 \\
HSA : MOXH $=1: 2.5$ & 55.1 & 7.8 & 15.6 & 25.2
\end{tabular}

observed both in peak I $(4 \mathrm{~nm})$ and peak II $(7 \mathrm{~nm})$. These findings indicated that binding with $\mathrm{MOXH}$ changed the conformation of HSA, and led to an decrease in polarity surrounding the Trp and Tyr residues.

3.4.4. CD spectroscopy. CD spectroscopy is a reliable and sophisticated method to analyze the secondary structure change of protein after ligand binding. ${ }^{37}$ The CD spectra of HSA in the absence and presence of MOXH exhibited two negative bands at $208 \mathrm{~nm}$ and $222 \mathrm{~nm}$ as shown in Fig. 3(c), which indicates the dominance of the typical $\alpha$-helix structure. ${ }^{24}$ The data were further analyzed by the algorithm SELCON $3,{ }^{38}$ and the fractions of $\alpha$-helix, $\beta$-sheet, turn, and unordered coil were listed in Table 4. The free HSA comprised $46.3 \% \alpha$-helix, $14.0 \% \beta$ sheet, $16.9 \%$ turn, and $25.4 \%$ unordered coil. After addition of MOXH, the $\alpha$-helix content increased whereas the $\beta$-sheet and

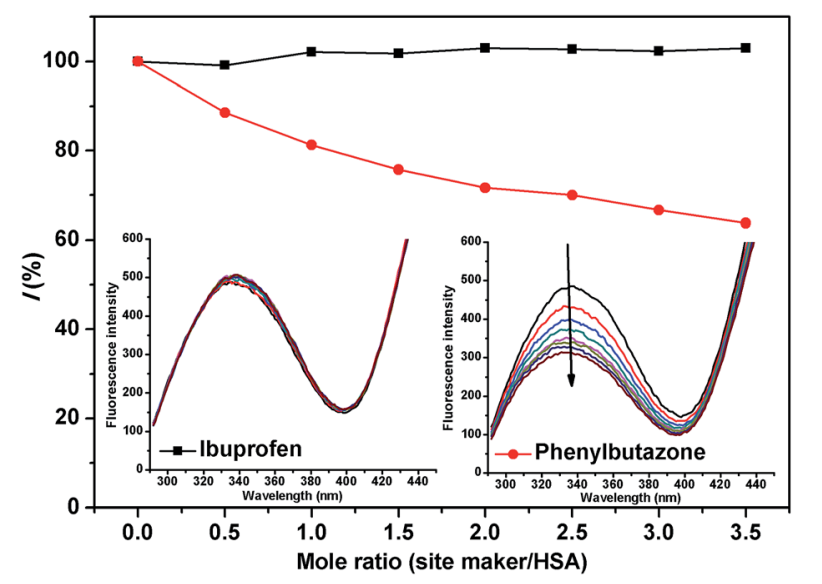

Fig. 5 Effect of site markers on the fluorescence of MOXH-HSA. Both $\mathrm{HSA}$ and MOXH concentrations were $2.0 \times 10^{-6} \mathrm{~mol} \mathrm{~L}^{-1}$, site markers concentrations varied from 0 to $7 \times 10^{-6} \mathrm{~mol} \mathrm{~L}^{-1}$. 


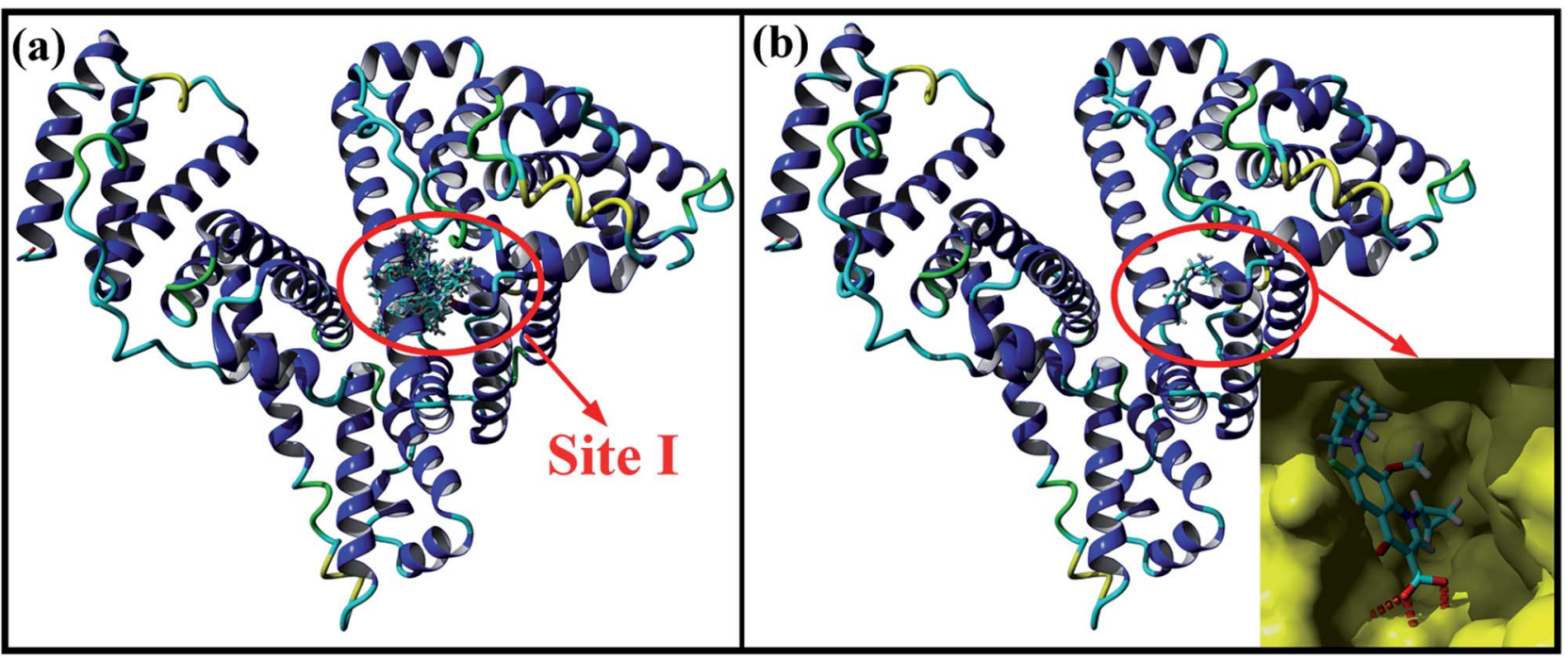

Fig. 6 (a) Cluster analysis for the first thirty conformations of MOXH binding to HSA. (b) The lowest energy conformation of the MOXH-HSA complex and the partial enlarged drawing of HSA site I in surface form.

turn content decreased. When the molar ratio of MOXH to HSA reached $2.5: 1$, the $\alpha$-helix, $\beta$-sheet and turn were changed to $55.1 \%, 7.8 \%, 15.6 \%$, respectively. Thus, the interaction of MOXH and HSA altered the secondary structure of HSA. And the original bonding of HSA, which is necessary to form secondary and tertiary protein structures, was affected when MOXH inserted into HSA.

\subsection{Site marker displacement studies}

HSA is a globular protein that contains three homologous helical domains (I, II, and III), and each domain can be divided into two subdomains (A and B). ${ }^{39}$ Crystal structure analysis indicated that there are two major and structurally selective binding sites called Sudlow site I and Sudlow site II in HSA, which are located in subdomains IIA and IIIA, respectively. ${ }^{\mathbf{4 0}}$

To investigate the possible binding sites of MOXH, site marker displacement studies were conducted using phenylbutazone and

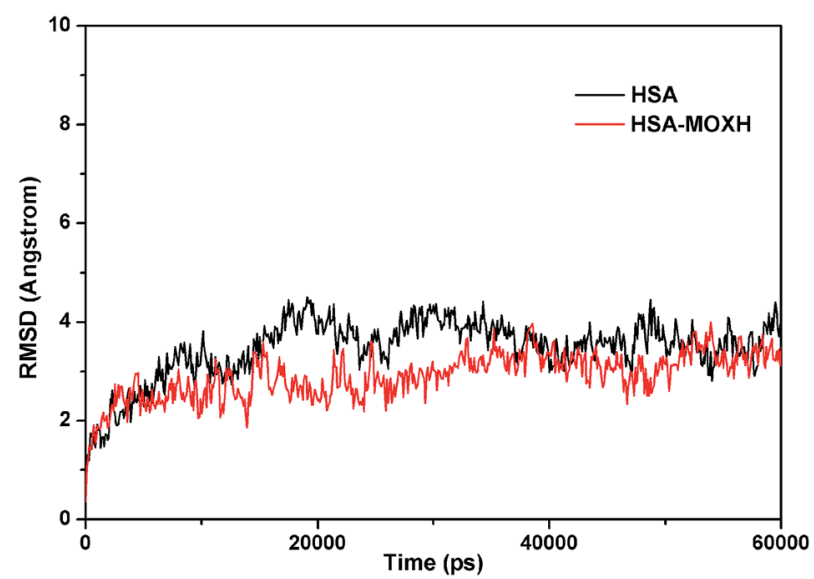

Fig. 7 RMSDs of the MD simulations for the free HSA and MOXH-HSA complex systems.
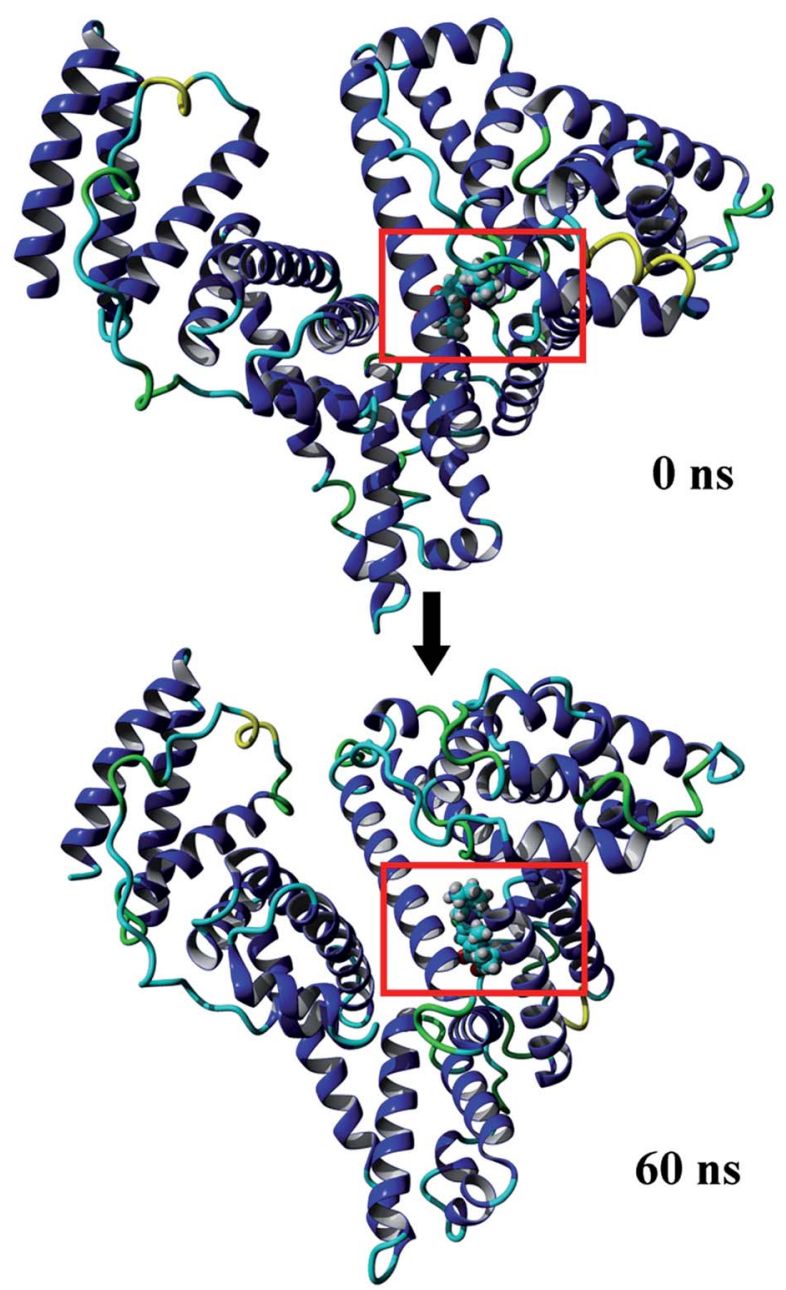

Fig. 8 Comparison between initial conformation of the MOXH-HSA complex and conformation of the complex at 60 ns. 
ibuprofen as sites I and II probes, respectively. ${ }^{\mathbf{4 1}}$ The percentage of displacement $(I)$ by a site marker can be calculated as follows: ${ }^{15}$

$$
I(\%)=\frac{F_{2}}{F_{1}} \times 100
$$

where $F_{2}$ and $F_{1}$ are the fluorescence intensities of MOXH-HSA in the presence and absence of site markers, respectively. The plot of $I$ values against the molar ratio of probe to HSA is shown in Fig. 5. The fluorescence intensity decreased remarkably with increasing phenylbutazone concentration, whereas a negligible effect was observed with ibuprofen addition. These results suggested that MOXH and phenylbutazone shared the same binding site thereby phenylbutazone competed with MOXH. Thus, MOXH was mainly bound to site I in subdomain IIA of HSA.

\subsection{Molecular docking studies}

Molecular docking studies provide some insights into the interaction between small molecule and biomacromolecule. In the present study, molecular docking was performed to further identify the exact binding site of MOXH on HSA and promote visual understanding of the binding site. As shown in Fig. 6(a), the first thirty binding conformations of MOXH obtained according to scores clustered in site I of HSA, which indicated that MOXH preferentially bound to HSA in site I. The lowest energy conformation was chosen to analyze the binding details [Fig. 6(b)]. The diazabicyclo in MOXH molecule inserted into the hydrophobic cavity of HSA in subdomain IIA. Residues Gln 196, Arg 257, His 288, Ala 291, and Glu 292 were involved in the hydrophobic interaction with MOXH [Fig. 9(a)] (left). Furthermore, the Glu 153, Arg 218, and Arg 222 residues formed four
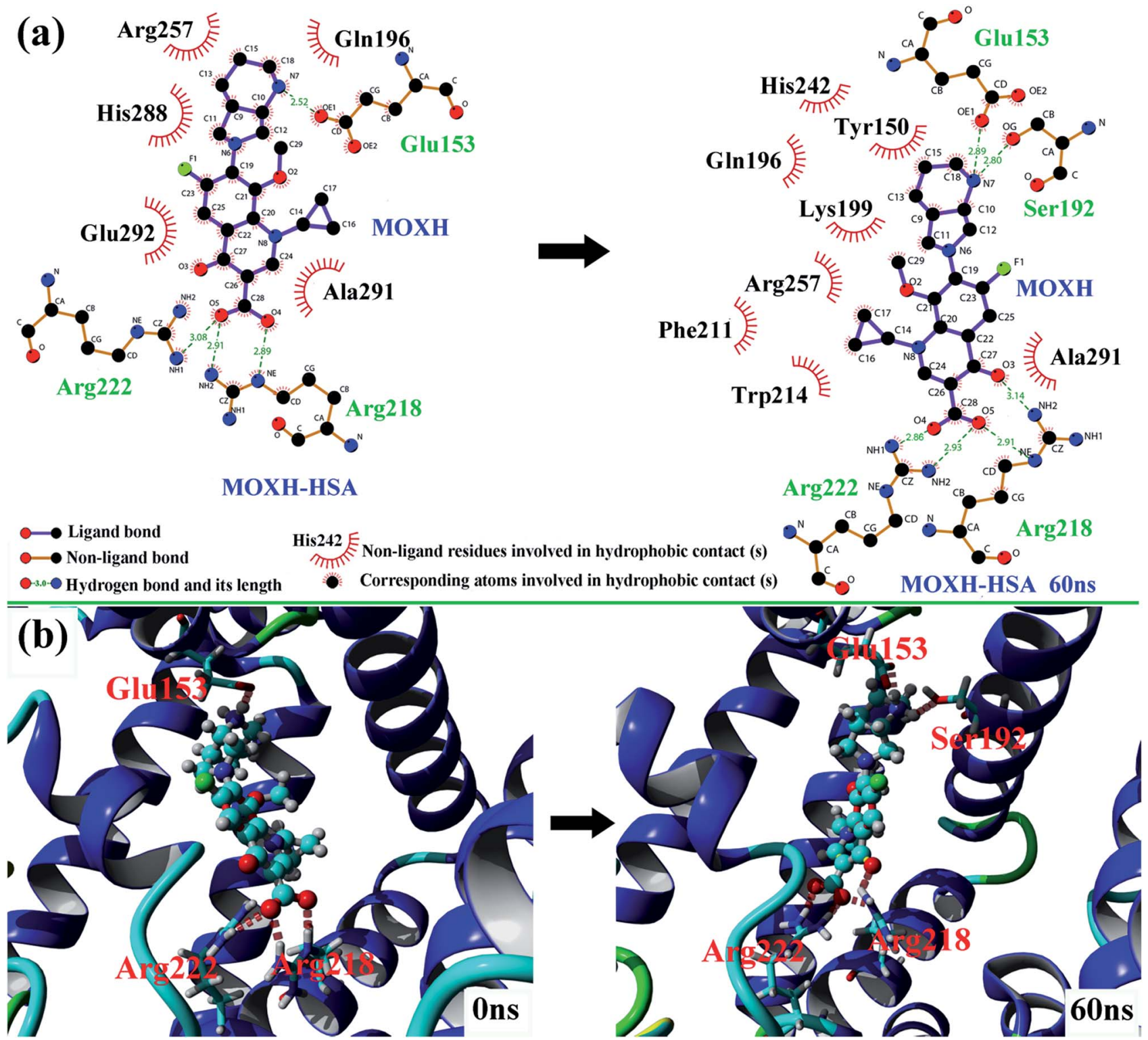

Fig. 9 (a) The two-dimensional maps of interactions before and after MD simulations of 60 ns were calculated using LigPlot. (b) Comparison of the hydrogen bonds between $\mathrm{MOXH}$ and residues before and after MD simulations of $60 \mathrm{~ns}$. 
hydrogen bonds with MOXH. The carboxyl of Glu 153 connected to the $\mathrm{NH}_{2}$ group of $\mathrm{MOXH}$ though a hydrogen bond with a length of $2.52 \AA$. The $\mathrm{NH}_{2}$ and $\mathrm{NH}$ groups of the Arg 218 side chain built two hydrogen bonds with carboxyl of $\mathrm{MOXH}$, at distances of $2.91 \AA$ and $2.89 \AA$, respectively. The $\mathrm{NH}_{2}$ group of the Arg 222 side chain formed a hydrogen bond with the carboxyl of MOXH (3.08 $)$. These results indicated that the hydrophobic force and hydrogen bonds played key roles in stabilizing the MOXH-HSA complex.

\subsection{MD simulations}

MD simulations were conducted to check out the stability of the HSA and MOXH-HSA complex systems. The structure stability of free HSA and MOXH-HSA were evaluated based on their root mean square deviation (RMSD) values. As shown in Fig. 7, the RMSD values of both systems were plotted and calculated against the simulation time scale of 0-60 ns. Results indicated that both systems reached equilibration and oscillated at around a value of $40 \mathrm{~ns}$. In addition, the MOXH remained in site I of HSA after $60 \mathrm{~ns}$, and both MOXH and HSA showed evident conformational changes (Fig. 8). Therefore, it is ascertained that MOXH can steadily bind to the site I of HSA. The MOXHHSA complex was stabilized after $40 \mathrm{~ns}$ due to conformational rearrangement. Further analysis revealed that two more hydrogen bonds formed after the conformational optimization of MD [Fig. 9(a)] (right). The enlarged three-dimensional maps of hydrogen bond interactions are shown in [Fig. 9(b)]. The length and position of the hydrogen bonds also changed to some extent. The $\mathrm{NH}_{2}$ group of MOXH formed two hydrogen bonds with the oxygen atoms of Glu 153 (2.89 ̊) and Ser 192 $(2.80 \AA)$, respectively. The $\mathrm{NH}_{2}$ and $\mathrm{NH}$ groups of Arg 218 built two hydrogen bonds with the carbonyl oxygen (3.14 $\AA$ ) and two hydrogen bonds with the carboxyl oxygen (2.91 ̊) of $\mathrm{MOXH}$, respectively. Two $\mathrm{NH}_{2}$ groups of Arg 222 were connected to the carboxyl oxygen atoms of MOXH through two hydrogen bonds with a distance of $2.93 \AA$ and $2.86 \AA$, respectively. More residues including Tyr 150, Gln 196, Lys 199, Phe 211, Trp 214, Arg 257, His 242, and Ala 291 were involved in the hydrophobic interaction with MOXH. In addition, the VDW force $(2.3 \times$ $10^{5} \mathrm{~kJ} \mathrm{~mol}^{-1}$ ) was also involved in the MOXH-HSA binding. In conclusion, hydrogen bonds and VDW force played major roles in stabilizing the MOXH-HSA complex, and hydrophobic force was also involved in the binding process.

\section{Conclusions}

Multi-spectroscopic methods and computational simulations were applied to explore the binding characteristics, interaction mode, and conformational changes of MOXH with HSA. The fluorescence spectroscopy demonstrated that MOXH interacted with HSA through the complex formation via static mechanism, which was proved by time-resolved fluorescence. According to fluorescence data, binding of MOXH to HSA was relatively strong with binding constants $\left(K_{\mathrm{a}}\right)$ of $10^{5} \mathrm{~L} \mathrm{~mol}^{-1}$. The calculated thermodynamic parameters indicated that hydrogen bonds and van der Waals force were the major elements to drive the MOXH-HSA binding. The UV-vis spectra, synchronous fluorescence, and 3D fluorescence results suggested that binding with MOXH changed the conformation of HSA, and increased the hydrophobicity around the Trp residues. The secondary structure of HSA was affected by the MOXH-HSA binding; an increase in $\alpha$-helix content whereas a decrease in $\beta$ sheet and turn content of HSA were induced in binding process, considering the $\mathrm{CD}$ data. Site marker displacement experiments revealed that MOXH mainly bound to site I in subdomain IIA of HSA. The molecular docking results suggested the site I binding mode, which coincides well with the experimental results. Molecular dynamics simulations further corroborated the reliability of the docking results, and provided a more reasonable conformation of the MOXH-HSA complex. Six hydrogen bonds formed between MOXH and residues (Glu 153, Ser 192, Arg 218, and Arg 222) of HSA, together with the VDW force and hydrophobic force, played major roles in stabilizing the MOXH-HSA complex. All these results indicated that MOXH could steadily bind to site I of HSA. This study might contribute to a better understanding of binding mechanism of MOXH with HSA, and help to comprehend the dynamic equilibrium process of drugprotein interaction.

\section{Conflicts of interest}

There are no conflicts to declare.

\section{Acknowledgements}

This work was supported by the Large-scale Science Instrument Shareable Platform Construction of Sichuan Province (Grant No. 2015JCPT0005-15010102) and the Large-scientific Instruments Sharing Platform Ability Construction of Sichuan Province (Grant No. 2016KJTS0037).

\section{References}

1 C. M. Culley, M. K. Lacy, N. Klutman and B. Edwards, Am. J. Health-Syst. Pharm., 2001, 58, 379-388.

2 Y. K. Lv, P. Li, M. L. Jiao, B. S. Liu and C. Yang, Turk. J. Chem., 2014, 38, 202-209.

3 B. S. Liu, C. Yang, X. N. Yan, J. Wang, J. S. Zhang and Y. K. Lü, Spectrosc. Lett., 2012, 45, 175-183.

4 M. Miravitlles and A. Anzueto, Expert Opin. Pharmacother., 2008, 9, 1755-1772.

5 Y. Chu, J. Qu, L. Y. Qu, Y. F. Luo and M. Y. Jiang, Drug Res., 2015, 65, 650-657.

6 A. Navalon, R. Blanc, L. Reyes, N. Navas and J. L. Vilchez, Anal. Chim. Acta, 2002, 454, 83-91.

7 B. T. Yin, C. Y. Yan, X. M. Peng, S. L. Zhang, S. Rasheed, R. X. Geng and C. H. Zhou, Eur. J. Med. Chem., 2014, 71, 148-159.

8 D. Wu, Y. Zhai, J. Yan, K. Xu, Q. Wang, Y. Li and H. Li, RSC Adv., 2015, 5, 11036-11042.

9 A. E. Radi, T. Wahdan, Z. Anwar and H. Mostafa, Electroanalysis, 2010, 22, 2665-2671. 
10 M. Rezaei-Tavirani, S. H. Moghaddamnia, B. Ranjbar, M. Amani and S. A. Marashi, BMB Rep., 2006, 39, 530-536.

11 P. Lee and X. Wu, Curr. Pharm. Des., 2015, 21, 1862-1865.

12 Z. Sun, H. Xu, Y. Cao, F. Wang and W. Mi, J. Mol. Liq., 2016, 219, 405-410.

13 A. Safarnejad, M. Shaghaghi, G. Dehghan and S. Soltani, J. Lumin., 2016, 176, 149-158.

14 S. Li, J. He, Y. Huang, Q. Wang, H. Yang, K. Xu and H. Li, RSC Adv., 2016, 6, 85811-85819.

15 B. Tang, Y. Huang, X. Ma, X. Liao, Q. Wang, X. Xiong and H. Li, Food Chem., 2016, 212, 434-442.

16 M. Rarey, B. Kramer, T. Lengauer and G. Klebe, J. Mol. Biol., 1996, 261, 470-489.

17 M. Rarey, S. Wefing and T. Lengauer, J. Comput.-Aided Mol. Des., 1996, 10, 41-54.

18 E. Krieger, T. Darden, S. B. Nabuurs, A. Finkelstein and G. Vriend, Proteins, 2004, 57, 678-683.

19 Y. Duan, C. Wu, S. Chowdhury, M. C. Lee, G. Xiong, W. Zhang, R. Yang, P. Clieplak, R. Luo, T. Lee, J. Caldwell, J. Wang and P. Kollman, J. Comput. Chem., 2003, 24, 19992012.

20 A. Jakalian, D. B. Jack and C. I. Bayly, J. Comput. Chem., 2002, 23, 1623-1641.

21 A. C. Wallace, R. A. Laskowski and J. M. Thomton, Protein Eng., 1995, 8, 127-134.

22 J. He, D. Wu, Y. Zhai, Q. Wang, X. Ma, H. Yang and H. Li, J. Mol. Liq., 2016, 219, 547-553.

23 X. Li, D. Chen, G. Wang and Y. Lu, Eur. J. Med. Chem., 2013, 70, 22-36.

24 X. Li and S. Wang, New J. Chem., 2015, 39, 386-395.

25 B. K. Bozoğlan, S. Tunç and O. Duman, J. Lumin., 2014, 155, 198-204.

26 J. Chamani, H. Vahedian-Movahed and M. R. Saberi, J. Pharm. Biomed. Anal., 2011, 55, 114-124.
27 M. R. Ajmal, N. Zaidi, P. Alam, S. Nusrat, M. K. Siddiqi, G. Badr, H. M. Mahmoud and R. H. Khan, J. Biomol. Struct. Dyn., 2017, 35, 46-57.

28 P. Mandal, M. Bardhan and T. Ganguly, J. Photochem. Photobiol., B, 2010, 99, 78-86.

29 G. Paramaguru, A. Kathiravan, S. Selvaraj, P. Venuvanalingam and R. Renganathan, J. Hazard. Mater., 2010, 175, 985-991.

30 P. D. Ross and S. Subramanian, Biochemistry, 1981, 20, 30963102.

31 C. Challier, P. Beassoni, C. Boetsch, N. A. Garcia, M. A. Biasutti and S. Criado, J. Photochem. Photobiol., B, 2015, 142, 20-28.

32 L. Tang, H. Zuo and L. Shu, J. Lumin., 2014, 153, 54-63.

33 Y. J. Hu, Y. Liu, Z. B. Pi and S. S. Qu, Bioorg. Med. Chem., 2005, 13, 6609-6614.

34 H. Tanzadehpanah, A. Asoodeh, H. Mahaki, Z. Mostajabodave, J. Chamani, Z. Mojallal-Tabatabaei, S. Emtenani and M. R. Moradi, Process Biochem., 2016, 51, 2067-2075.

35 B. Tu, Z. F. Chen, Z. J. Liu, R. R. Li, Y. Ouyang and Y. J. Hu, RSC Adv., 2015, 5, 73290-73300.

36 Z. Chen, H. Xu, Y. Zhu, J. Liu, K. Wang, P. Wang, S. Shang, X. Yi, Z. Wang, W. Shao and S. Zhang, RSC Adv., 2014, 4, 25410-25419.

37 P. Alam, S. K. Chaturvedi, T. Anwar, M. K. Siddiqi, M. R. Ajmal, G. Badr, M. H. Mahmoud and R. Hasan Khan, J. Lumin., 2015, 164, 123-130.

38 N. Sreerama and R. W. Woody, Anal. Biochem., 2000, 287, 252-260.

39 B. Ekman, T. Sjödin and I. Sjöholm, Mol. Pharmacol., 1979, 16, 767-777.

40 A. Divsalar and S. Khodabakhshian, J. Mol. Liq., 2015, 206, 82-88.

41 H. Chen, H. Rao, P. He, Y. Qiao, F. Wang, H. Liu, M. Cai and J. Yao, Environ. Sci. Pollut. Res., 2014, 21, 11340-11348. 